\title{
Strates
}

STRATES Matériaux pour la recherche en sciences sociales

$10 \mid 2001$

Villageois et citadins de Grèce

\section{L'automobile en Grèce : à la source d'une nouvelle relation entre villes et villages}

\section{Michel Sivignon}

\section{(2) OpenEdition \\ 1 Journals}

Édition électronique

URL : http://journals.openedition.org/strates/32

DOI : $10.4000 /$ strates.32

ISSN : $1777-5442$

Éditeur

Laboratoire Ladyss

Édition imprimée

Date de publication : 1 mai 2001

ISSN : 0768-8067

Référence électronique

Michel Sivignon, "L'automobile en Grèce : à la source d'une nouvelle relation entre villes et villages », Strates [En ligne], 10 | 2001, mis en ligne le 11 janvier 2005, consulté le 08 septembre 2020. URL :

http://journals.openedition.org/strates/32 ; DOI : https://doi.org/10.4000/strates.32

Ce document a été généré automatiquement le 8 septembre 2020

Tous droits réservés 


\title{
L'automobile en Grèce : à la source d'une nouvelle relation entre villes et villages
}

\author{
Michel Sivignon
}

Le point de vue que je veux donner ici est celui d'un observateur étranger attentif qui regarde la Grèce depuis plus de trente-cinq ans.

2 Il y a deux visions de l'espace rural dans nos pays européens. Dans la première, notre société et l'espace dans lequel elle s'inscrit sont totalement urbanisés et il existe désormais une continuité entre la ville et la campagne telle que la notion d'espace rural n'a pas de sens et que l'adjectif rural n'a plus de valeur scientifique pour une étude de la société contemporaine. Les modes de vie et les valeurs se sont totalement uniformisés et la ville est la seule référence.

3 La Grande-Bretagne peut en Europe représenter l'extrême de cette évolution, avec une part des agriculteurs dans la population active très réduite et un paysage qui n'est plus qu'une scenery pour les urbains.

4 Mais on peut aussi, à l'inverse, considérer que l'espace rural continue, à travers les faibles densités et l'éloignement des centres urbains, à garder des caractéristiques propres et qu'il n'est pas seulement une portion du territoire national desséchée et abandonnée.

5 Je ne souhaite pas entrer dans une discussion sans issue, mais participer au débat en m'en tenant à un fil directeur concret, l'usage de l'automobile comme moyen de transformation de l'espace rural et des relations entre ville et campagne durant les trente-cinq dernières années.

6 La conquête de l'espace grec par l'automobile est certainement un des faits géographiques majeurs survenus dans la seconde moitié du XXe siècle. En quelques dizaines d'années, la Grèce est passée d'une situation où la possession d'une automobile est un privilège rare à une « automobilisation » généralisée de la société, rurale comme urbaine. 
7 Les progrès de l'équipement automobile n'ont pas été seulement une amélioration technique ; ils ont modifié les rapports que les Grecs entretiennent avec leur territoire et les relations que la ville entretient avec la campagne s'en sont trouvées métamorphosées. Les statistiques nous disent qu'en 1962 on dénombrait 56000 automobiles de tourisme en Grèce. Trente-cinq ans plus tard soit le temps d'une génération, le chiffre est passé à 2500000 . Une répartition géographique de l'automobile montre que désormais la campagne ne souffre plus, dans ce domaine, d'aucun retard sur la ville. Bien davantage, la généralisation de l'usage de l'automobile dans les régions les plus difficiles d'accès a permis parfois de « remobiliser » les terroirs autrefois abandonnés.

La circulation automobile dans la Grèce de 1950

81950 marque la fin de la guerre civile, d'où la Grèce sort exsangue. Les voies de communication sont très affectées par le conflit et globalement, les routes carrossables étant fort rares, l'essentiel des transports se fait par le rail et par la mer.

9 Pourtant la Grèce n'a jamais connu d'âge d'or du chemin de fer. Elle a reçu du XIX ${ }^{\mathrm{e}}$ siècle un réseau squelettique, réduit à quelques lignes. Rappelons que le réseau à voie normale se compose de la ligne d'Athènes à Salonique et de la ligne de Salonique à la frontière turque de Thrace. Les relations internationales sont assurées en quatre points : deux avec la Yougoslavie, un avec la Bulgarie et un avec la Turquie. À quoi il faut ajouter quelques voies secondaires comme celle qui relie Chalcis à la voie AthènesSalonique. En outre une voie ferrée à écartement métrique fait le tour du Péloponnèse. Les voyageurs étrangers qui circulent en Grèce empruntent ces voies ferrées et les transports maritimes. Il faut se reporter aux descriptions pittoresques du géographe Jules Sion en 1930 quand il prépare sa description de la Grèce de la Géographie universelle de Paul Vidal de la Blache et Lucien Gallois (Armand Colin, 1930). Jules Sion connaît surtout de la Grèce continentale ce qui est accessible en chemin de fer. Parlant de l'Arcadie dans le centre du Péloponnèse, il précise : « Le centre de la péninsule possède maintenant un réseau de routes carrossables. À vrai dire, elles sont peu nombreuses et mettent à une dure épreuve les automobiles qui viennent y terminer leur carrière ; un chauffeur grec 'boit l'obstacle' aussi aisément que l'ouzo, l'anisette de là-bas; il n'hésite pas à prendre les raccourcis pierreux des piétons sur des pentes raides, à suivre un lit de torrent et, si le pneu tombe en lambeaux, il le remplace en tordant une brassée de cistes ou de bruyères. Mais il vaut la peine d'emprunter ses services ou ceux de muletiers pour visiter les montagnes d'Arcadie.» («Dans Le Péloponnèse », Bulletin de la Société Languedocienne de Géographie, $1934,2^{\circ}$ et $3^{\circ}$ fascicule, p. 63).

Ce réseau ferré embryonnaire avait beaucoup souffert pendant la guerre civile et antérieurement pendant la seconde guerre mondiale. Il était facile de détruire les multiples ouvrages d'art qui parsemaient son tracé. Et les nombreuses casemates qui l'accompagnent encore de nos jours nous disent ce que furent les affrontements entre ceux qui s'efforçaient de maintenir le trafic et leurs adversaires.

11 Le réseau routier n'était pas en meilleure situation et on ne s'étonnera pas que l'essentiel du trafic intérieur se fit par voie maritime. Comme au XIX ${ }^{e}$ siècle le caïque sur la mer et la mule sur le sentier empierré ou caldérimi étaient les moyens de transport privilégiés.

12 Les charrois sur des charrettes tirées par des chevaux ou des bœufs étaient l'exception sauf dans les plaines, comme en Thessalie où en 1950 encore on rencontrait des chars à roue pleine identiques à ceux que décrit l'archéologue Heuzey en 1856. Il résultait de 
cette situation qu'il y avait peu de circulation de marchandises à longue distance, que les déplacements étaient longs et les tonnages transportés réduits. À une échelle plus modeste, celle des communautés rurales, l'accès aux différents éléments du finage était problématique. Les bergers mettaient plusieurs heures pour parvenir aux pâturages de montagne, même si ces estives étaient incluses dans les limites de la commune. Ces difficultés et la présence encore générale des loups rendaient indispensable qu'ils dorment auprès de leurs troupeaux pendant de longues semaines. Dans les régions spécialisées dans la culture de l'olive (le Pélion par exemple), la longueur des transports à l'intérieur même de la commune rendait indispensable l'habitat double: chaque famille avait une maison dans le bourg et une ou plusieurs kalyvia, sorte de mazet assez vaste cependant pour qu'une famille pût y demeurer pendant plusieurs mois, de novembre à mars, saison de la cueillette.

Une carte de la circulation automobile en Grèce éditée par le ministère des Travaux Publics en 1956 donne une image frappante du morcellement des transports : les routes ne sont fréquentées que dans un périmètre restreint autour de chaque petite ville et les convois à longue distance sont exceptionnels. Une seule voie carrossable permet le passage routier entre la Thessalie et l'Epire: celle de la passe de Katara (1700 m d'altitude), commandée par la petite ville de Metsovo.

14 L'absence de réseau urbain, autrement dit d'une hiérarchie urbaine articulée, s'expliquait largement par cette difficulté des communications. À une échelle plus modeste, celle de nos chefs-lieux de canton, de toutes petites bourgades jouissaient en dépit de leur taille réduite d'une activité commerciale non négligeable : l'émiettement est la règle. On a noté que dans le domaine maritime le caïque est le pendant du mulet. Sans doute sa capacité de transport était-elle plus importante que celle d'une caravane : quelques tonnes ou quelques dizaines de tonnes selon la taille. Le faible tirant d'eau du caïque lui donnait accès à de modestes rades foraines. À cette époque, on se mit à couler du béton dans quelques fûts de tôle pour construire à bon compte un appontement perpendiculaire au rivage. Le moteur diesel s'était substitué à la voile latine et de multiples lignes de navigation locale desservaient tous les littoraux. Là encore, la dispersion de ces ports multiples était bien accordée à la petite taille des agglomérations qui les accompagnent.

Naissance d'un réseau routier national

La fin de la guerre civile et les effets du plan Marshall amenèrent un effort particulier dans le domaine du réseau routier. Pendant la guerre civile elle-même et pour des besoins militaires on s'était efforcé de multiplier les routes. La liaison entre événements militaires et développement routier est d'ailleurs ancienne en Grèce : les soldats du général Maison s'y étaient employés en 1829, entre Kalamata et la mer, et, à partir de 1917, le corps expéditionnaire français avait entrepris la construction de quelques bonnes routes comme celle qui partant d'Itéa franchit le col de Gravia et permet de relier le Golfe de Corinthe à celui de Lamia.

La médiocrité du réseau ferré et son incapacité à résoudre les problèmes de circulation de la Grèce continentale firent que l'essentiel des investissements se porta sur la route. L'orientation fut encore plus nette à partir des années soixante lorsque Constantin Caramanlis arriva au pouvoir. La route devint le signe même de la modernité. La création d'un vrai réseau routier supposait que l'on construisît quelques voies à grande circulation pour relier les grandes villes. La grande affaire fut la construction de la route nationale numéro 1 menant d'Athènes à Salonique. On choisit de construire une 
sorte de voie express à péage dont les caractéristiques techniques permirent de supporter le trafic pendant une vingtaine d'années. Elle avait en outre l'avantage d'un tracé moins montagneux que celui de la voie ferrée du XIX ${ }^{e}$ siècle car on ne redoutait plus comme premier danger les bombardements à partir des cuirassés croisant dans la mer Egée. Certaines régions d'accès difficile comme la plaine d'Atalandi, le long du golfe de l'Eubée (Evoïkos Kolpos) furent désenclavées et purent se spécialiser dans la production de légumes à destination d'Athènes.

17 La construction d'un réseau de routes goudronnées profita au premier chef à un type de villes que l'on peut définir comme des villes moyennes intérieures vivant principalement des relations avec leur arrière-pays agricole. C'était le cas de Larissa par rapport à Volos, ville maritime favorisée dans la phase précédente. Conformément à ce modèle figurent Serres et Drama par rapport à Kavala, Agrinion par rapport à Missolonghi, Arta par rapport à Prévéza. On peut dire que d'une façon globale les villes agricoles, centres de régions que l'irrigation amenait à un développement rapide, enregistrèrent des progrès plus vifs que les organismes portuaires qui subissaient durement les effets de la concentration des trafics dans le port du Pirée : la route l'emportait désormais sur la voie maritime.

Dans le même temps, tous les chefs-lieux de département de la Grèce continentale, même les plus excentrés, furent reliés à Athènes et secondairement à Salonique par des lignes d'autobus à grande fréquence. La plus remarquable initiative fut la constitution, à diverses échelles spatiales (celle de la région, celle du département, et même parfois celle de l'aire de chalandise d'une petite ville) de coopératives de propriétaires d'autobus (dites KTEL), fédérés et bénéficiaires d'un système de lignes organisées par les pouvoirs publics. Dans ce domaine, la route et l'automobile jouèrent un rôle essentiel dans l'organisation du territoire national.

À une échelle plus modeste, celle des départements, ou nomos, la route permit pour la première fois de relier commodément les villages aux chefs-lieux. Dans un premier temps, jusque vers 1965 , la totalité des villes de plus de 5000 habitants fut reliée au monde extérieur par une route asphaltée. Dans le même temps, les villages étaient desservis par des chemins de terre empierrés mais carrossables et, vers 1970, chacune des 6000 communes de la Grèce, ou presque, était accessible à l'automobile. Là encore, à l'échelle du département, une bonne organisation de lignes d'autobus permit de relier tous les jours toutes les communes à leur chef-lieu, soulignant le rôle de l'automobile dans l'intégration du monde rural à l'espace national en termes économiques sans doute, mais aussi en termes de relations sociales et d'accès aux équipements scolaires et de santé.

La révolution automobile

20 La construction et le développement de ce réseau routier vont de pair avec un accroissement très rapide du nombre des véhicules. Le nombre total des véhicules en circulation s'élevait à 146000 en 1962, dont un peu plus du tiers en véhicules de tourisme, et le reste en camions, autobus, motocyclettes, etc. En 1978 on est passé à 1166000 véhicules dont 744000 véhicules de tourisme, et en 1997 à 4048000 véhicules dont 2500000 automobiles de tourisme. On remarquera que la proportion des automobiles de tourisme s'est accrue, indice d'une élévation certaine du niveau de vie. L'équipement en véhicules ne présente plus de différence sensible si on le rapporte au chiffre de la population, ce qui est la signe d'une sorte de saturation. Saturation bien 
relative puisque la Grèce est encore loin du degré d'équipement automobile des pays de l'Europe occidentale.

21 Si le développement privilégié du réseau routier a pu apparaitre comme une forme d'encouragement au développement de l'automobile, ce dernier a été contrarié par une politique fiscale qui taxe lourdement les automobiles de tourisme. En revanche la Grèce a très tôt encouragé la possession de véhicules professionnels à l'usage des commerçants, des artisans et des agriculteurs: les véhicules professionnels sont dispensés des lourdes taxes à l'achat qui frappent les véhicules de tourisme. Ces privilèges ont amené un accroissement considérable des véhicules dits agrotiko c'est-àdire à usage agricole. Tout agriculteur peut posséder une fourgonnette et l'utiliser dans un certain périmètre autour de son exploitation agricole. Le contrôle de cette limitation se fait grâce à l'inscription du nom et de la raison sociale du propriétaire sur la carrosserie du véhicule. Dans les régions de plaine, le développement de l'agrotiko n'a pas empêché celui du tracteur parce que l'agrotiko ne peut être utilisé pour les diverses façons culturales et qu'un motoculteur de petite taille éventuellement transportable sur le plateau d'une camionnette n'est pas adapté à la grande culture (céréales, coton, betteraves à sucre, etc.). Dans les bonnes plaines irriguées, on constate donc à la fois la multiplication du tracteur et l'usage généralisé de l'agrotiko. Dans certaines régions, comme les régions productrices d'olives où l'agriculteur ne possède qu'un petit troupeau de chèvres ou moutons à usage familial, où les outils de culture sont de petite taille (la tronçonneuse pour l'élagage), et où la plate-forme de la camionnette suffit pour transporter la cueillette journalière d'olives, l'agrotiko est le grand outil de modernisation de la campagne. Ajoutons que lorsque bon nombre d'agriculteurs exercent une autre profession (le plus souvent dans la construction), l' agrotiko est une fois encore le bienvenu. Inversement les ânes et les mulets ont quasiment disparu. Là où une heure de trajet à dos de mulet était nécessaire pour aller élaguer des arbres, dix minutes de voiture suffisent et la charge de bois transportable est beaucoup plus importante. On ne s'étonnera pas que le développement de l'agrotiko ait abouti à un véritable reclassement des terroirs ruraux dans ces régions d'oliveraies pris à titre d'exemple. Seuls sont désormais cultivés, engraissés et élagués les arbres d'un accès facile en agrotiko. Les autres tendent à être progressivement abandonnés. Ou du moins sont-ils traités de loin en loin : l'olive qui était un produit de culture devient en quelque sorte un produit de cueillette.

On peut toutefois repérer des évolutions en sens opposé. Dans la Grèce centrale, la grande culture spéculative de ces dernières années a été le coton. Le coton procurait de tels revenus que chacun tentait d'en semer n'importe où. Le développement des chemins vicinaux, de simples pistes ouvertes au bulldozer, a amené un accès facile à des bouts de champs dont il suffisait qu'ils soient à peu près plans pour qu'on y fasse passer un tracteur et qu'on puisse les arroser. On observe donc des défrichements qui étendent l'espace cultivé dans l'espoir d'y récolter du coton.

Dans les régions qui disposent de vastes terrains de parcours, ces derniers ont été équipés de pistes carrossables. Dans cet effort d'équipement, les fonds de l'Union européenne ont joué un rôle décisif. Les bergers qui se situaient au dernier degré de la hiérarchie sociale parce qu'ils étaient contraints de passer la nuit avec leurs brebis pendant la saison d'été ont vu leur mode de vie amélioré de façon décisive grâce à la desserte de leur bergerie d'altitude au moyen de l'agrotiko. Ce véhicule leur permet de 
rentrer au village chaque soir, d'apparaitre sur la place publique, de vivre cette vie urbaine qu'affectionnent tous les Grecs, même ceux des villages.

Un nouveau mode d'habiter

On entre par le biais de cet exemple dans le chapitre des changements décisifs du mode d'habiter: mode d'habiter le village et mode aussi de concevoir le rapport entre le village et la ville voisine. La très grande facilité des communications, la multiplication et la généralisation de l'automobile ont dans une certaine mesure mis fin à la malédiction villageoise et ne justifient plus guère l'opinion péjorative que les Grecs ont du village, un lieu où il ne se passe jamais rien et où la vie de société est embryonnaire. Ces transformations sont particulièrement visibles dans les régions de plaine où la prospérité apportée par telle culture (en Thessalie le coton, sur la côte nord du Péloponnèse les agrumes) transforme un pays, qui correspondrait à peu près à une de nos sous-préfectures, en un réseau de petites villes et de villages proches où l'avantage d'habiter les premières n'est plus décisif. Soit l'éparchie ou sous-préfecture de Farsala en Thessalie. Les villages périphériques des hauteurs se sont presque vidés de leur population permanente. La seule culture est celle du blé dur qui permet de récolter du grain mais plus encore des subventions de Bruxelles. Bon nombre d'agriculteurs ont choisi de résider en appartement à Farsala et viennent en voiture cultiver leurs champs. Dans la plaine, au contraire, on remarque des maisons neuves parfois luxueuses dans les villages fortement enrichis grâce aux revenus élevés que procure le coton. Ici, l'avantage d'habiter en ville, surtout quand il s'agit d'une petite ville de 10000 habitants comme Farsala, n'est plus incontestable. Le territoire de la souspréfecture est sous-tendu par un réseau de villages d'accès facile où se dispersent les usines d'égrenage de coton, les ateliers de réparation de machines agricoles, les représentations des marques de tracteurs ou de produits phytosanitaires. Le centre de soins de Farsala et son lycée sont aisément accessibles en moins d'une demi-heure de voiture. La notion même d'urbain et de rural perd de son sens. Depuis longtemps déjà, l'abandon de l'élevage au profit des cultures annuelles caractérisées par le rythme saisonnier très marqué des travaux agricoles, permet un habitat double, surtout pour les plus fortunés des agriculteurs. Les femmes et les enfants d'âge scolaire résident en ville et, pour ses agriculteurs, le village devient seulement un lieu de travail. Dans le même temps, des non-agriculteurs qui, par héritage, disposent au village de quelque terrain à bâtir, habitent au village et travaillent en ville. Toutefois ce dernier cas n'est pas le plus courant.

Depuis 1980 surtout, des bourgs de petite taille, qui sont l'équivalent de l'un de nos chefs-lieux de canton de 2000 habitants, ont reçu un remarquable équipement en centres de soins primaires et en lycées. Or, on observe que l'accès à l'automobile conduit les médecins de ces centres et les professeurs de ces lycées à habiter de préférence dans la ville la plus proche.

Dans d'autres régions, particulièrement les régions littorales où le tourisme balnéaire s'est considérablement développé (on mentionne ici aussi bien la Grèce continentale que la Grèce insulaire), les repères ordinaires du rural et de l'urbain sont encore plus difficiles à situer. La voiture permet en effet de partager sa semaine entre l'appartement en ville et les chambres d'hôtes sur le littoral que l'on vient gérer et surveiller. Nombreuses sont les familles qui résident à la ville en hiver et à la plage en été. La proximité de la ville d'une part, l'usage de la voiture de l'autre, permettent des 
modes de vie mixtes. Il en résulte un nouveau statut des littoraux qui se trouvent urbanisés même s'ils ne sont pas situés dans une ville.

Conclusion

Dans cette Grèce profondément transformée, l'organisation du territoire oppose plutôt aujourd'hui les campagnes urbanisées grâce à la proximité de la ville et à l'usage de la voiture, et les campagnes de ce que nous appellerions le rural profond dont beaucoup sont en voie d'abandon. Ces transformations ont été soutenues par l'étonnante prospérité de certaines régions agricoles. En Thessalie, du point de vue des revenus et de la ponction fiscale, il vaut mieux être cultivateur de coton que fonctionnaire. La prospérité des agriculteurs se traduit dans le luxe de l'habitation et l'agrotiko devient un $4 \mathrm{X} 4$ rutilant. Il est vrai que bien des fonctionnaires, d'origine rurale proche, empochent eux aussi les dividendes du progrès agricole en louant leurs terres aux membres de la famille restés à la ferme.

Dans ces métamorphoses, l'automobile n'est pas le moteur principal. Mais la généralisation de son usage induit les transformations profondes de la vie relationnelle et amène de nouveaux modes d'appropriation du temps et de l'espace.

\section{RÉSUMÉS}

La conquête de l'espace grec par l'automobile est un fait géographique majeur. Les progrès extrêmement rapides de l'équipement n'ont pas seulement conduit à des améliorations techniques, ils ont aussi modifié les rapports que les Grecs entretiennent avec leur territoire et les relations de la ville et de la campagne.

À l'échelle locale, la construction d'un réseau de routes praticables en toute saison date des années soixante. Ce fut un instrument important d'intégration du monde rural à l'espace national en termes économiques, mais aussi en termes de relations sociales et d'accès aux équipements scolaires et de santé.

Dans le même temps qu'elle taxait lourdement les véhicules de tourisme, la Grèce a très tôt encouragé la possession de véhicules professionnels. L'usage généralisé de fourgonnettes, dites en grec agrotiko, a révolutionné la vie du village et a permis aux agriculteurs d'exercer une autre profession. Leur multiplication a en outre facilité, grâce à des pistes taillées au bulldozer, la mobilisation de finages abandonnés depuis longtemps. Elle a autorisé l'extension des cultures de bon rapport financier, comme le coton. Elle a totalement changé la condition des bergers, qui peuvent aujourd'hui rentrer au village chaque soir.

On observe enfin l'émergence de nouveaux modes d'habiter: des agriculteurs ont un appartement en ville et se rendent chaque jour au village. Inversement, les villages situés à une distance raisonnable d'une ville retiennent une population de non-agriculteurs. Ici comme ailleurs, l'opposition entre villes et campagnes est une notion dont les termes sont à revoir.

The conquest of Greek space by the automobile is a major geographic event. Rapid progress in equipment has not only lead to technical advances but also to change in the types of connections that the Greeks maintain with their territory and the relations between towns and the countryside. 
At the local level, the network of roads accessible in all seasons was established in the 1960s. It served as an important instrument in the integration of rural areas into national economic activity, as well as in terms of social relations and access to education and health services.

Whilst imposing heavy taxes on tourist vehicles, Greece encouraged possession of professional vehicles from early on. General use of small vans, called agrotiko in Greek, transformed village life and allowed farmers to carry out other activities. The increase in number of these vans, along with tracks laid down with bulldozers, helped stimulate previously abandoned areas. It allowed for expansion in the production of rentable crops such as cotton. It completely transformed conditions for shepherds, who were able to return to their village every night.

Lastly, there is evidence of emerging new modes of living: agricultural producers will have an apartment in town and go to the village every day. Conversely, those villages located at a reasonable distance from town will contain an amount of non-agricultural professionals. Here, as elsewhere, the opposition between towns and countryside is a notion which is in need of revision.

\section{INDEX}

Index géographique : Grèce

Mots-clés : Rural/urbain

\section{AUTEUR}

MICHEL SIVIGNON

Ladyss, professeur émérite de l'université Paris X-Nanterre 